\title{
The Muon g-2 Experiment at Fermilab
}

\author{
Martin Fertl ${ }^{a, *}$ \\ ${ }^{a}$ Institute of Physics, Johannes Gutenberg University Mainz, \\ Staudinger Weg 7, 55128 Mainz, Germany \\ E-mail: mfertl@uni-mainz.de
}

The E989 collaboration has recently published the most precise measurement of the muon anomalous magnetic moment $a_{\mu}$ with an uncertainty of $0.46 \mathrm{ppm}$. The new experimental world average of $a_{\mu}$ deviates by 4.2 standard deviations from the Standard Model prediction provided by the Muon g-2 Theory Initiative. To extract the value of $a_{\mu}$ a frequency comparison experiment is performed with spin-polarized muons confined in a superbly controlled electric and magnetic field environment. A measurement of the difference between the Larmor and the cyclotron frequency, the so-called anomalous spin precession frequency, is combined with a high-precision measurement of the magnetic field environment which is performed using nuclear magnetic resonance techniques. I discussed the most recent result of the Fermilab Muon g-2 experiment's first physics data run performed in 2018. I reported on the experimental improvements implemented for the subsequent science runs that will allow the collaboration to achieve the ultimate goal of $0.14 \mathrm{ppm}$ uncertainty on $a_{\mu}$.

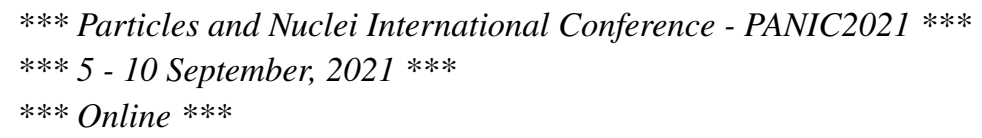

${ }^{*}$ Speaker 


\section{Introduction}

A particle's magnetic moment $\vec{\mu}$ is related to its spin $\vec{s}$ through the gyromagnetic factor $g$

$$
\vec{\mu}=g \frac{q e}{2 m} \vec{s},
$$

with $q$ and $m$ representing the particle's charge (in units of the elementary charge) and mass. Fusing special relativity and quantum mechanics, Dirac predicted in 1928 that for a point-like charged lepton $g=2$ [1]. The first experimental significant deviation from this prediction was found by Kusch and Foley in 1947 for the electron [2] and explained by Schwinger through the addition of the lowest order radiative correction term of quantum electrodynamics (QED) [3]. The deviation from the Dirac prediction defines the anomalous magnetic moment

$$
a=\frac{g-2}{2}=a_{\mathrm{QED}}+a_{\mathrm{EW}}+a_{\mathrm{Had}}+a_{\mathrm{BSM}}
$$

which includes contributions from QED, electroweak, hadronic physics as well as possible BeyondStandard-Model physics. For the muon, the most precise measurement of $a_{\mu}$ was previously performed by the Muon g-2 experiment at Brookhaven National Laboratory (BNL E821), $a_{\mu}^{\mathrm{BNL}}=$ $116592089(63) \times 10^{-11}$, in the early 2000's with a relative uncertainty of $0.54 \mathrm{ppm}$. The latest prediction of $a_{\mu}$ was published by the Muon g-2 Theory Initiative, $a_{\mu}^{\text {pred }}=116591810(43) \times 10^{-11}$, with a relative precision of $0.37 \mathrm{ppm}$ [5], in 2020. The uncertainty budget of the prediction is dominated by the contributions related to hadronic physics, hadronic vacuum polarization and hadronic light-by-light scattering. This discrepancy between the BNLE821 result and the theoretical prediction motivates the Muon g-2 experiment at Fermi National Accelerator Laboratory (FNAL E989) to determine $a_{\mu}$ with a factor-four-smaller relative uncertainty to $0.14 \mathrm{ppm}$ [6].

\section{Experimental principle}

The Muon g-2 experiment extracts $a_{\mu}$ from a measurement of the anomalous spin precession frequency $\omega_{a}$ and of the magnetic field $B$ experienced by the muons. In a homogeneous magnetic field (no electric fields) a relativistic muon can be viewed as carrying two intrinsic clocks related to its momentum and its spin. Positive muons $(q=+1)$ will perform cyclotron motion with the angular frequency

$$
\vec{\omega}_{\mathrm{c}}=\frac{e}{m \gamma} \vec{B},
$$

where $\gamma$ is the relativistic Lorentz factor and $\vec{B}$ the magnetic flux density vector. The coupling of the magnetic field to the muon's magnetic moment causes spin precession with angular frequency

$$
\vec{\omega}_{\mathrm{s}}=-g \frac{e}{2 m} \vec{B}-(1-\gamma) \frac{e}{\gamma m} \vec{B}
$$

The difference between the angular frequencies is independent of the muon's momentum,

$$
\vec{\omega}_{\mathrm{a}}=\vec{\omega}_{\mathrm{s}}-\vec{\omega}_{\mathrm{c}}=-a \frac{e}{m} \vec{B} .
$$

In this idealized situation the longitudinal polarization of the muon, $P_{\mathrm{L}}=\frac{\vec{s} \cdot \vec{p}}{|\vec{s}||\vec{p}|}$, changes at the rate of $\omega_{\mathrm{a}}$. Transverse electrostatic fields are employed to confine muons in the storage ring up to a certain transverse momentum. Then $P_{\mathrm{L}}$ changes according to 


$$
\frac{d}{d t} P_{\mathrm{L}}=\frac{d}{d t}(\hat{\beta} \cdot \vec{s})=-\frac{e}{m} \vec{s}_{\perp} \cdot\left[a_{\mu} \hat{\beta} \times \vec{B}+\left(a_{\mu}-\frac{1}{\gamma^{2}-1}\right) \beta \vec{E}\right],
$$

with $\hat{\beta}=\vec{\beta} /|\vec{\beta}|, \vec{s}_{\perp}$ the spin component perpendicular to $\hat{\beta}$ and $\vec{E}$ the electric focusing field. The first term in rectangular brackets includes the non-relativistic and homogenous magnetic field limit. The second term reflects the effects of the relativistically generated magnetic fields, the electric focusing field and the transverse muon motion which result in the well-known "electric field" and "pitch" corrections. The FNAL E989 experiment follows the approach of previous experiments to use muons of 'magic' momentum $p=3.094 \mathrm{GeV} / \mathrm{c}(\gamma=29.3)$ to cancel the electric field related contributions to first order.

To extract the numerical value of $a_{\mu}$ the Muon g-2 experiment determines $\omega_{a}$ as well as the volume and time averaged magnetic flux density $\tilde{B}$

$$
a_{\mu}=\frac{\omega_{\mathrm{a}}}{\tilde{B}} \frac{m_{\mu}}{e}=\frac{\omega_{\mathrm{a}}}{\tilde{\omega}_{\mathrm{p}}^{\prime}\left(T_{\mathrm{r}}\right)} \frac{\mu_{\mathrm{p}}^{\prime}\left(T_{\mathrm{r}}\right)}{\mu_{\mathrm{e}}(H)} \frac{\mu_{\mathrm{e}}(H)}{\mu_{\mathrm{e}}} \frac{m_{\mu}}{m_{\mathrm{e}}} \frac{g_{\mathrm{e}}}{2} .
$$

The angular frequency $\tilde{\omega}_{\mathrm{p}}^{\prime}\left(T_{\mathrm{r}}\right)$ is the time- and volume- averaged equivalent frequency of protons in a spherical cell of water at the reference temperature $T_{\mathrm{r}}$ which is related to the magnetic field through a product of the highly precisely known ratios of the magnetic moment of protons in a spherical sample of water to the magnetic moment of the electron bound in an hydrogen atom, $\frac{\mu_{\mathrm{p}}^{\prime}\left(T_{\mathrm{r}}\right)}{\mu_{\mathrm{e}}(H)}$ [7], the ratio of the magnetic moment of the electron in a hydrogen atom to the magnetic moment of the bare electron, $\frac{\mu_{\mathrm{e}}(H)}{\mu_{\mathrm{e}}}$ [8], the ratio of the masses of muons and electrons, $\frac{m_{\mu}}{m_{\mathrm{e}}}$ [9], as well as the g-factor of the free electron, $\frac{g_{\mathrm{e}}}{2}$ [4]. The total uncertainty contribution to $a_{\mu}$ from the tying with the external ratios is $24 \mathrm{ppb}$.

\section{Measured quantities of the Muon g-2 experiment}

The Muon g-2 experiment determines the frequency ratio $\frac{\omega_{\mathrm{a}}}{\tilde{\omega}_{\mathrm{p}}^{\prime}\left(T_{\mathrm{r}}\right)}$. The ratio itself includes corrections and calibration factors to take the experimental conditions into account. The full ratio,

$$
R^{\prime}=\frac{\omega_{\mathrm{a}}}{\tilde{\omega}_{\mathrm{p}}^{\prime}\left(T_{\mathrm{r}}\right)}=\frac{f_{\text {clock }} \omega_{\mathrm{a}}^{\text {meas }}\left(1+C_{\mathrm{e}}+C_{\mathrm{p}}+C_{\mathrm{ml}}+C_{\mathrm{pa}}\right)}{f_{\text {calib }}\left\langle M(x, y, \phi) \omega_{\mathrm{p}}^{\prime}(x, y, \phi)\right\rangle\left(1+B_{\mathrm{k}}+B_{\mathrm{q}}\right)},
$$

includes the measured anomalous spin precession frequency $\omega_{\mathrm{a}}^{\text {meas }}$ corrected for the systematic frequency biases related to the electric field $C_{\mathrm{e}}$, the pitching muon motion $C_{\mathrm{p}}$, the loss of muons $C_{\mathrm{ml}}$ and the calorimeter phase acceptance effect $C_{\mathrm{pa}}$. The clocks of the data acquisition system for the $\omega_{a}$-determination were detuned by a blinding factor $f_{\text {clock }}$ which was only revealed to the collaboration after all the internal data (analysis) consistency tests were passed. In the denominator, $f_{\text {calib }}$ represents the calibration factor that ties the readings of the magnetic field measurement devices to the absolute magnetic field standard. The convolution, $\langle\ldots\rangle$, accounts for the overlap of the spatial muon $M(x, y, \phi)$ and magnetic field $\omega_{\mathrm{p}}^{\prime}(x, y, \phi)$ distributions. The distribution $M(x, y, \phi)$ is reconstructed using two positron tracker stations in combination with extensive muon beam dynamics modeling. A map $\omega_{\mathrm{p}}^{\prime}(x, y, \phi)$ is determined every two to three days and subsequent maps are interpolated in time with information obtained from an array of pNMR magnetometers 
installed in the walls of the vacuum chambers surrounding the muon storage region. The correction terms $B_{\mathrm{k}}$ and $B_{\mathrm{q}}$ account for transient magnetic fields related to the injection of the muon beam into the storage ring, which are absent during the determination of the map $\omega_{\mathrm{p}}^{\prime}(x, y, \phi)$.

\subsection{Muon beam}

The time structure of the $8 \mathrm{GeV}$-proton beam hitting the production target of Fermilab's muon campus (two times eight bunches with a short (long) time separation of $10 \mathrm{~ms}$ ( $200 \mathrm{~ms}$ ) is optimized to avoid event pile-up in the experiment's detectors. The debris emerging from the target is focused with a pulsed magnetic lens into a momentum selective beam line which transports positive particles of momentum around $3.1 \mathrm{GeV}$. In the two-body decay of positive pions a highly polarized beam of muons is generated. Each 120-ns-long beam pulse is cleaned from remaining hadronic impurities (protons, pions, ...) in the energy-dispersive delivery ring such that a purely leptonic beam is injected into the storage ring magnet.

\subsection{Muon storage ring}

The muon beam enters into the gap of a C-shaped magnet through a bore in the iron backbone of the magnet. A super-conducting magnet locally compensates the return magnetic field such that the muons travel on a straight path. The vertical magnetic field of $1.45 \mathrm{~T}$ bends the muons onto a circular orbit with radius $R=7.12 \mathrm{~m}$. Without further manipulation the muon beam would hit the backside of the inflector magnet at the end of its first revolution around the ring. To transfer the beam onto a storable orbit a series of three fast magnetic kickers is employed. To confine the beam in vertical direction a system of four electro-static quadrupoles (ESQ) is installed around $43 \%$ of the muon storage region, a $90 \mathrm{~mm}$ minor-diameter torus in the center of the vacuum chambers installed in the $180 \mathrm{~mm}$-high pole gap of the magnet.

\subsection{Anomalous spin precession frequency}

The angular frequency $\omega_{a}$ is directly measurable in the laboratory. Parity violation in the muon rest frame causes a preferred emission of high-energy decay positrons along the direction of the muon spin. The emission direction modulation relative to the muon momentum results in a laboratoryframe modulation of the kinetic energy of the in-spiraling decay positrons which are detected in one of 24 electromagnetic calorimeters equidistantly distributed along the inner circumference of the storage ring. The Cherenkov light generated by each positron is detected by silicon photomultipliers ( $\mathrm{SiPMs}$ ) attached to the 54 individual $\mathrm{PbF}_{2}$ crystals of a calorimeter station and the resulting light signal trace is digitized at the rate of the blinded data acquisition system frequency. To avoid a systematic frequency pull the energy calibration of the calorimeters has to be known to the $10^{-4}$ level. A dedicated laser calibration system [15] has been implemented to achieve this stability on all time scales of the experiment, nanoseconds (beam injection) to years (the data taking phase of the experiment). Decay positrons above a certain energy threshold are then registered according to their arrival time in a histogram, see Figure 1a, which shows an amplitude modulation with the dominant frequency $\omega_{a}^{\text {meas }}$. The data set for Run-1 was collected in four different subsets of storage ring parameter settings. Three different schemes were applied to reconstruct the positron kinetic energies and six independent groups performed eleven analyses to extract $\omega_{a}$. The total uncertainty of $\omega_{a}$ is dominated by the statistical uncertainty contribution of $434 \mathrm{ppb}$. 


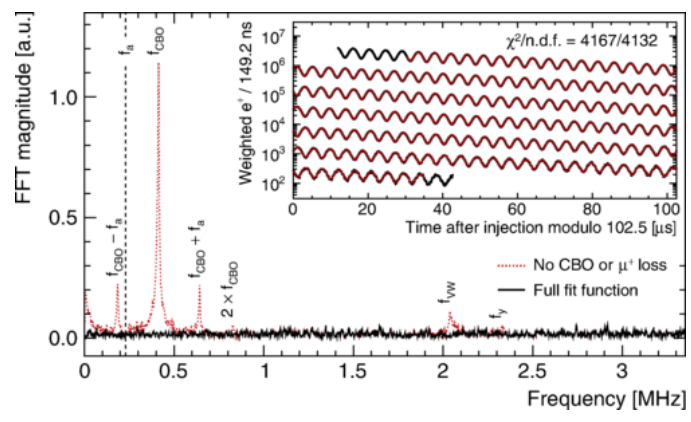

(a)

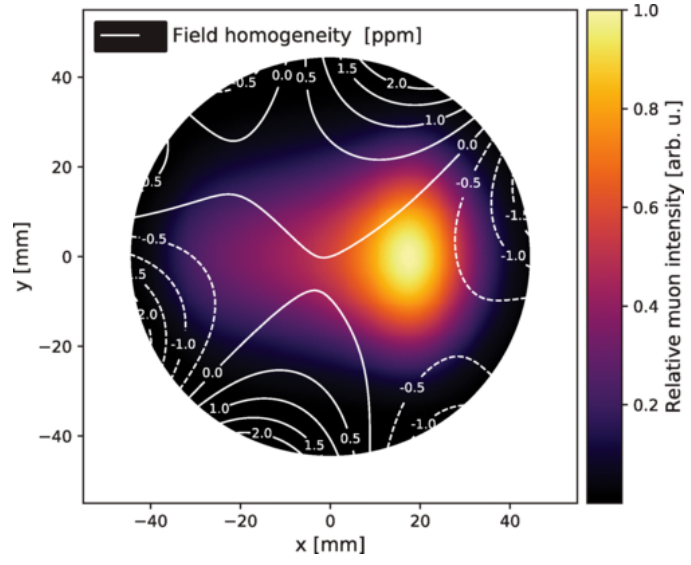

(b)

Figure 1: (a) Fourier transform of residuals of a fit to the arrival time histogram (inset) with (black) and without (red) accounting for the complex muon beam dynamics. Figure from [10]. (b) Azimuthally averaged muon beam distribution overlaid onto the azimuthally averaged magnetic field map.

\subsubsection{Electric field and pitch corrections}

The measured value of $\omega_{\mathrm{a}}^{\text {meas }}$ has to be corrected for frequency shifts induced by the muon beam motion in the field of the electrostatic quadrupoles (ESQ). By symmetry, the field of the ESQ only vanishes at their center. As a consequence of the radial offset of the muon distribution from the geometrical ESQ center the so-called electric field correction needs to be applied. The vertical motion of the muon beam in the ESQ field results in the so-called pitch correction. These two corrections, $C_{\mathrm{p}}=180(13) \mathrm{ppb}$ and $C_{\mathrm{e}}=489(53) \mathrm{ppb}$, are the largest systematic correction to $\omega_{\mathrm{a}}^{\text {meas }}$.

\subsubsection{Phase acceptance effect and lost muons}

The exact arrival time of an individual positron at a calorimeter is influenced by the individual parent-muon's spin orientation and a decay-position-dependent time of flight. The histogram results from the average over the muon beam ensemble. Due to a hardware difficulty concerning the charging of two plates of the ESQ system a time-dependent electric field induced a time-dependence of the muon beam position and width. This effect was eliminated for subsequent data taking runs but a large correction of $C_{\mathrm{pa}}=-158(75) \mathrm{ppb}$ had to be applied for this first dataset. Furthermore, a momentum-correlated muon spin orientation combined with a momentum-correlated muon loss rate from the ring can induce an early-to-late pull of the ensemble-averaged spin orientation and bias $\omega_{a}^{\text {meas }}$. A small correction of $-11(5)$ ppb was applied.

\subsection{Magnetic field measurement chain}

The determination of the magnetic field is as important as the determination of $\omega_{\mathrm{a}}$. As the operation of the muon beam is mutually exclusive with the determination of the magnetic field distribution a combination of a spatial and temporal interpolation of the magnetic field is used to extract the magnetic field the muons experience when circulating in the ring. The magnetometers employed to determine the spatial magnetic field distribution are based on pNMR and the individual probes are 
regularly calibrated relative to an absolute magnetic field probe based on a very well characterized water sample before and after a data set run.

\subsubsection{Spatial magnetic field distribution}

The spatial magnetic field distribution in the muon storage region is determined typically every two to three days. The so-called trolley contains 17 pNMR probes arranged in two concentric rings (with one probe at the center) and measures at about 9000 azimuthal positions around the ring. Averaged around the ring magnetic field variations of $1 \mathrm{ppm}$ are typically observed across the muon storage aperture. The spatial distribution is expressed in a multipole expansion.

\subsubsection{Temporal interpolation}

During muon beam operation a fixed-position array of 378 pNMR probes is used to track the temporal evolution of the magnetic field. The probes are located on top and below the muon storage volume, in the walls of the vacuum chambers, and are organized in 72 stations. Time-instabilities of the magnetic field are caused e.g. by changes of the experimental hall's temperature. These induce in particular a diurnal modulation of the radial gradient of the magnetic flux density as the gap of the $\mathrm{C}$-shaped magnet opens and closes. The average dipole field around the ring is stabilized by active feedback regulation of the current in the magnet coils.

\subsubsection{Convolution of muon and magnetic field distribution}

The spatial distribution of the muon beam $M(x, y, \phi)$ is reconstructed from the spatial distribution of the decay positrons detected in two straw-tracker stations in combination with extensive beam simulations. To determine the magnetic field experiences by the muon ensemble $M(x, y, \phi)$ is convoluted with the magnetic field map $\omega_{\mathrm{p}}^{\prime}(x, y, \phi)$, indicated in equation 8 by $\langle\ldots\rangle$. The azimuthally averaged muon beam distribution is overlaid onto the azimuthally averaged magnetic field map in figure $1 \mathrm{~b}$. The convolution procedure including the absolute calibration was performed with a relative uncertainty of $56 \mathrm{ppb}$.

\subsubsection{Transient magnetic fields}

The trolley-measured magnetic field maps need to be corrected for two transient magnetic fields related to the muon beam operation. The first correction is related to the kicker magnet system that transfers the muon beam onto its storable orbit. Eddy currents are induced in the vacuum chamber walls and generate a beam-related magnetic field contribution over a time scale of the first $2 \mu \mathrm{s}$. The size of the effect was measured with two dedicated Faraday magnetometers and a correction to the magnetic field map $\tilde{\omega}_{\mathrm{p}}$ of $B_{\mathrm{k}}=-27(37) \mathrm{ppb}$ was applied. The second correction is related to the ESQ system. Despite its name, the electric field is only static on the time scale of a single muon bunch injection and is dropped in between to reduce the number of high-voltage discharges caused by trapped electrons. The repeated charging of the ESQ plates before every beam pulse causes mechanical vibrations of the conductors which again cause a perturbation of the magnetic field. This effect was only revealed with dedicated measurements after Run 2 and a correction with a very conservative uncertainty estimate was applied to Run 1 data, $B_{\mathrm{q}}=17(92) \mathrm{ppb}$. 


\section{New Muon g-2 result}

The result of the blinded analysis of the Run 1 data set of the Muon g-2 experiment provides the most precise individual measurement of the anomalous magnetic moment of the positive muon

$$
a_{\mu}(\text { FNAL }, \text { Run } 1)=116592040(54) \times 10^{-11}
$$

with a statistics-dominated total relative uncertainty of $0.46 \mathrm{ppm}$ [10]. A detailed discussion of the systematic effects mentioned here can be found in [11-13]. The new experimental result is in good agreement with the BNL E821 experiment and thus confirms this previous finding. As both results are statistically limited and independent of each other we provide new world average value

$$
a_{\mu}(\operatorname{Exp})=116592061(41) \times 10^{-11}
$$

with a total relative uncertainty of $0.35 \mathrm{ppm}$. The value $a_{\mu}(\operatorname{Exp})$ deviates from the SM prediction $a_{\mu}^{\text {pred }}=116591810(43) \times 10^{-11}$ by 4.2 standard deviations $(\sigma)$. The largest uncertainty contribution to the SM prediction is related to the so-called leading-order hadronic vacuum polarization contribution $a_{\mathrm{HVP}, \mathrm{LO}}^{\mathrm{SM}}=6931(40) \times 10^{-11}$. A new era in the field of Muon g-2 is opened up by the improved experimental result in combination with the rapid progress of lattice quantum chromo dynamics (LQCD) calculations assessing hadronic physics related contributions to $a_{\mu}$. Shortly after the release of the FNAL E989 result a LQCD-based prediction of the hadronic-vacuum-polarization contribution was published [14], $a_{\mathrm{HVP}, \mathrm{LO}}^{\mathrm{LQCD}}=7075(55) \times 10^{-11}$.

\section{Summary and Outlook}

The first result of the Muon g-2 experiment at FNAL provides the most precise measurement of the anomalous magnetic moment of the muon to date and confirms the findings of the BNL E821 experiment. The world average value shows a $4.2 \sigma$ tension with the SM prediction. The Muon g-2 experiment collected a data set of about 13 times the size of the BNL data set through a total of four data taking periods through July 2021. Improvements of the experimental conditions include the optimization of the kicker magnet strength which brings the muon beam onto its nominal storage orbit. A much better thermal stability of the experimental hall and the additional installation of isolation blankets around the iron yoke have increased the magnetic field stability. In addition, further dedicated measurements of the transitional magnetic fields related to the kickers and the ESQ system will allow to reduce the associated experimental uncertainties further. The Muon g-2 collaboration continues data collection since November 2021. Independently of the FNAL E989 experiment a complementary approach using an ultra-cold muon beam without the need for electrostatic focusing will be pursued at JPARC. To provide more input data for the SM prediction a new experiment, MuonE, has been proposed at CERN. The intense scrutiny of the first hadronic physics related LQCD calculation will be crucial to assess the significance of the persistent discrepancy as the experimental uncertainty will be further reduced to $140 \mathrm{ppb}$.

\section{Acknowledgments}

The Muon g-2 experiment was performed at the Fermi National Accelerator Laboratory, a U.S. Department of Energy, Office of Science, HEP User Facility. Fermilab is managed by Fermi 
Research Alliance, LLC (FRA), acting under Contract No. DE-AC02-07CH11359. Additional support for the author was provided by the German Research Foundation (DFG) through the Cluster of Excellence PRISMA+ (EXC 2118/1, Project ID 39083149).

\section{References}

[1] Dirac P. , The Quantum Theory of the Electron, Proc. R. Soc. London A 117: 610-624, 1928

[2] Kusch, P. and Foley, H. M., Precision Measurement of the Ratio of the Atomic ' $g$ Values' in the ${ }^{2} P_{\frac{3}{2}}$ and ${ }^{2} P_{\frac{1}{2}}$ States of Gallium, Phys. Rev. 72, 1256, 1947

[3] Schwinger J., On Quantum-Electrodynamics and the Magnetic Moment of the Electron, Phys. Rev. 73, 416, 1948

[4] Hanneke, D., et al., New Measurement of the Electron Magnetic Moment and the Fine Structure Constant, Phys. Rev. Lett. 2008, 100, 120801

[5] Aoyama, T., et al, The anomalous magnetic moment of the muon in the Standard Model, Physics Reports 887 (2020) 1-166

[6] Grange, J., et al, Muon (g-2) Technical Design Report, arXiv:1501.06858, 2015

[7] Phillips W. et al, Magnetic Moment of the Proton in $\mathrm{H} 2 \mathrm{O}$ in Bohr Magnetons, Metrologia 13 179,1977

[8] Mohr P., et al, CODATA 2014, Rev. Mod. Phys. 88, 035009 (2016)

[9] Liu W., et al, High Precision Measurements of the Ground State Hyperfine Structure Interval of Muonium and of the Muon Magnetic Moment, Phys. Rev. Lett. 82, 711, 1999

[10] Abi B., et al, Measurement of the Positive Muon Anomalous Magnetic Moment to $0.46 \mathrm{ppm}$, Phys. Rev. Lett. 126, 141801, 2021, https://doi.org/10.1103/PhysRevLett.126.141801

[11] Albahri T, et al., Measurement of the anomalous precession frequency of the muon in the Fermilab Muon g-2 Experiment, Phys. Rev. D 103, 072002, 2021, https://doi.org/10.1103/PhysRevD.103.072002

[12] Albahri T., et al., Magnetic-field measurement and analysis for the Muon g-2 Experiment at Fermilab, Phys. Rev. A 103, 042208, 2021, https://doi.org/10.1103/PhysRevA.103.042208

[13] Albahri T., et al., Beam dynamics corrections to the Run-1 measurement of the muon anomalous magnetic moment at Fermilab, Phys. Rev. Accel. Beams 24, 044002, 2021, https://doi.org/10.1103/PhysRevAccelBeams.24.044002

[14] S. Borsanyi, et al., Leading hadronic contribution to the muon magnetic moment from lattice QCD, Nature 593, 51-55 (2021)

[15] A. Anastasi, et al., The laser-based gain monitoring system of the calorimeters in the Muon g-2 experiment at Fermilab, JINST 14 P11025, 2019 